\title{
Study of the growth mechanisms of low-pressure chemically vapour deposited silica films
}

\author{
F. Ojeda, R. Cuerno*, R. Salvarezza** and L. Vázquez \\ Instituto de Ciencia de Materiales de Madrid, CSIC, Cantoblanco, 28049 Madrid, Spain \\ * Departamento de Matemáticas y Grupo Interdisciplinar de Sistemas Complicados, \\ Universidad Carlos III de Madrid, Av. Universidad 30, 28911 Leganés, Spain \\ ** INIFTA, Sucursal 4, Casilla de Correo 16, 1900 La Plata, Argentina
}

\begin{abstract}
We have studied the surface morphology evolution of $\mathrm{SiO}_{2}$ films grown at $20 \mathrm{~nm} / \mathrm{min}$ in a low-pressure chemical vapour deposition reactor from $\mathrm{SiH}_{4} / \mathrm{O}_{2}$ mixtures at low $(611 \mathrm{~K})$ and high $(723 \mathrm{~K})$ temperatures. Films have been deposited for times ranging from $10 \mathrm{~min}$ up to 48 hours. It is shown that the $\mathrm{SiO}_{2}$ growth at high temperature becomes stable, whereas at low temperature it is unstable (i.e., the surface roughness increases continuously with deposition time). This clear difference is explained on the basis of the different growth mechanisms operating under both experimental conditions. These results are compared with the predictions of the few theoretical works on growth evolution by chemical vapour deposition.
\end{abstract}

\section{INTRODUCTION}

Presently, the growth of solid films with high surface smoothness and uniformity is a stringent requirement in important industrial applications such as the manufacture of solid-state and optical devices. It is obvious that the possibility to control the structure and the properties of materials will require a fundamental level understanding of the materials growth processes. The main impediment to advances in this direction is that the growth and deposition techniques used in materials processing, particularly chemical vapour deposition (CVD), involve far from equilibrium kinetic processes which are not well understood. For this reason, the knowledge of the key parameters controlling the morphological evolution of growing films is a subject of increasing relevance in materials science.

CVD of silicon oxides from the $\mathrm{SiH}_{4} / \mathrm{O}_{2}$ reaction is a common growth process in the manufacture of silicon and III-V semiconductor-based electronic devices. This is mainly due to the capability of the $\mathrm{SiH}_{4} / \mathrm{O}_{2}$ reaction for achieving reasonable deposition rates at relatively low temperatures (typically between $300^{\circ}$ and $450^{\circ} \mathrm{C}$ ), which obeys to the abundant formation of highly reactive free radicals in the gas-phase [1,2]. Apart from this beneficial effect, the highly reactive character of the $\mathrm{SiH}_{4} / \mathrm{O}_{2}$ system results in poor step coverage of micro-sized trenches (non-conformal deposition) [3]. Moderation of $\mathrm{SiH}_{4} / \mathrm{O}_{2}$ reaction by addition of certain radical scavengers (e.g., $\mathrm{C}_{2} \mathrm{H}_{2}$ ) [4] or substitution of $\mathrm{SiH}_{4}$ by metalorganic precursors [5,6], mainly TEOS, are possible solutions to improve the step coverage conformality. Production of conformal high-quality $\mathrm{SiO}_{2}$ films from the $\mathrm{SiH}_{4} / \mathrm{O}_{2}$ reaction is also possible through a properly reactor design and choice of the process parameters [7,8]. However, the determining factor in film conformality is still a matter of debate, being reemission $[9,10]$ and surface mobility $[11]$ of the film precursor species the most accepted mechanisms.

In this work we study by atomic force microscopy (AFM) the morphological evolution of $\mathrm{CVD} \mathrm{SiO}_{2}$ films grown on silicon substrates. Film roughness variation with deposition time is examined within a very long temporal window in order to study the film growth stability. Spectral analysis of the AFM images is also performed to obtain information about the growth mechanisms and the length scales at which they operate. Results and their implications in the step-coverage problem are discussed on the basis of different growth mechanisms proposed in the literature.

\section{EXPERIMENTAL}

Silicon oxide $\left(\mathrm{SiO}_{2}\right)$ amorphous films were chemically vapour deposited on monocrystalline (100) oriented silicon wafers (Virginia Semiconductors). The deposition system was a low-pressure hot-wall 
horizontal CVD reactor. This reactor was fabricated from a quartz tube with an inner diameter of $6.1 \mathrm{~cm}$ and a total length of $100 \mathrm{~cm}$. The length of the heating zone, which is centred in a $30 \mathrm{~cm}$ long cylindrical furnace, is $10 \mathrm{~cm}$. A horizontal graphite plate was used as substrate-holder. The flow of the reactants, $\mathrm{SiH}_{4}$ (diluted at $2 \%$ in $\mathrm{N}_{2}, 99.999 \%$ purity) and $\mathrm{O}_{2}(99.9992 \%$ purity), was adjusted by means of mass flow controllers. Low-pressure conditions were achieved by means of a vacuum system that consists of a rotary pump boosted by a Roots pump. The process pressure was measured by a capacitative gauge and controlled by means of an electronically driven throttle valve. The thickness of the resulting $\mathrm{SiO}_{2}$ films was measured by means of a profilometer (Dektak 3030).

AFM measurements were performed with a Nanoscope III system (from Digital Instruments, Santa Barbara, CA) operating in tapping mode. Images, consisting in $512 \times 512$ data points, were taken at ambient conditions with silicon cantilevers. From the AFM images the surface roughness of the film can be obtained directly. Also, from the spectral analysis of them the surface roughness lateral correlations can be studied. For this purpose, we have obtained the Power Spectral Density (PSD) of the surface, defined by $\operatorname{PSD}(\mathbf{k}, t)=\left(4 \pi^{2} /^{2}\right) \cdot<h(\mathbf{k}, t) h(-\mathbf{k}, t)>$, where $L$ is the scan size and $h(k, t)$ the Fourier transform of $h(r, t)$, the height of the surface at time $t$ on the position $\mathbf{r}$ on the bidimensional substrate. The surface roughness, $\sigma$, can be shown to equal the square root of the wave-vector integral of $\operatorname{PSD}(k, t)$. For many growing surfaces $\sigma$ increases with time as $\sigma \propto \mathrm{t}^{\beta}$. Also, the PSD changes with the wavelength, $\lambda$, as PSD $\propto$ $(1 / \lambda)^{-(2+2 \alpha)}$. $\alpha$ and $\beta$, known as the roughness and growth exponents, respectively, describe the system dynamics. There are different growth models, described by continuum equations, which predict different sets of values for these exponents depending on the different growth mechanisms operating during the growth process [12].

\section{RESULTS}

The morphological study of CVD-grown $\mathrm{SiO}_{2}$ films has been focused on series of samples prepared at low $(611 \mathrm{~K})$ and high $(723 \mathrm{~K})$ temperatures for deposition times ranging from $10 \mathrm{~min}$ up to 48 hours. The deposition rate, which is an important growth parameter, is kept at $20 \mathrm{~nm} / \mathrm{min}$ in both cases. To this end, total gas flow rates of 50 and $250 \mathrm{sccm}$ were used for experiments performed at 723 and $611 \mathrm{~K}$, respectively. For both temperatures, total pressure was maintained at 1.4 Torr and $\left[\mathrm{O}_{2}\right]:\left[\mathrm{SiH}_{4}\right]$ ratio at 20 . Film thickness was observed to increase linearly with deposition time with the slope of $20 \mathrm{~nm} / \mathrm{min}$ mentioned above.

Figure 1 shows typical AFM images of the $\mathrm{SiO}_{2}$ films grown for 1 and $30 \mathrm{~h}$ at low (a,b) and high (c,d) temperature. Note the two different image areas taken for the $1 \mathrm{~h}\left(1 \times 1 \mu \mathrm{m}^{2}\right)$ and $30 \mathrm{~h}\left(14 \times 14 \mu \mathrm{m}^{2}\right)$ films. It is evident the surface roughening for both experimental conditions. The surface morphology is characterised, at low temperature, by a small granular structure with a typical size of $200-300 \mathrm{~nm}$ and a much larger, cauliflower-like, structure. This wider structure grows with time, in height and width, and determines the interface roughness at long length scales. On the other hand, the high temperature surface morphology is characterised at short length scales by a similar small granular structure $(200-300 \mathrm{~nm}$ in size). However, together with this structure a smaller granular structure (around $50 \mathrm{~nm}$ in size) appears scattered on the surface. This structure could be due to the formation in the gas phase of small silica clusters by gas-phase nucleation, which are finally deposited on the growing film surface. At large length scales also large structures can be detected but, this time, they do not resemble so much the cauliflower-like structures found at $611 \mathrm{~K}$.

Height profiles corresponding to deposition times ranging from 35 minutes up to 2 days are shown in Figure 2 for low (a) and high (b) temperature. At high temperature, the height profile seems to reach a stationary regime beyond $15 \mathrm{~h}$ of deposition, whereas at low temperature the surface roughening process seems to be still operating. This behaviour is better observed in Figure 3, where surface roughness appears as a function of deposition time at both temperatures. At low temperature, surface roughness increases continuously with deposition time. By contrast, at high temperature, although initially surface roughness increases with deposition time faster than at low temperature, it finally reaches a saturation regime around $30 \mathrm{~nm}$ for deposition times $>15 \mathrm{~h}$, as previously inferred from the height profiles.

PSD functions obtained from AFM images corresponding to samples grown for $48 \mathrm{~h}$ at low and high temperature are represented in Figure 4. The flat region of the spectra found for high wavelengths 
$611 \mathrm{~K}$
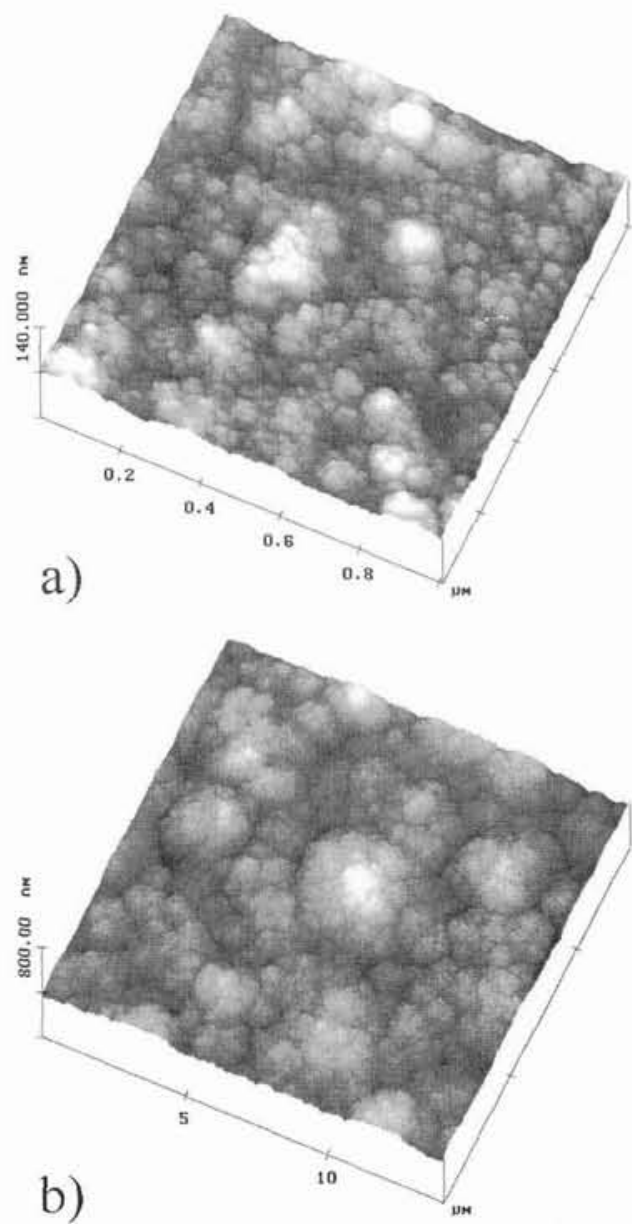

$723 \mathrm{~K}$
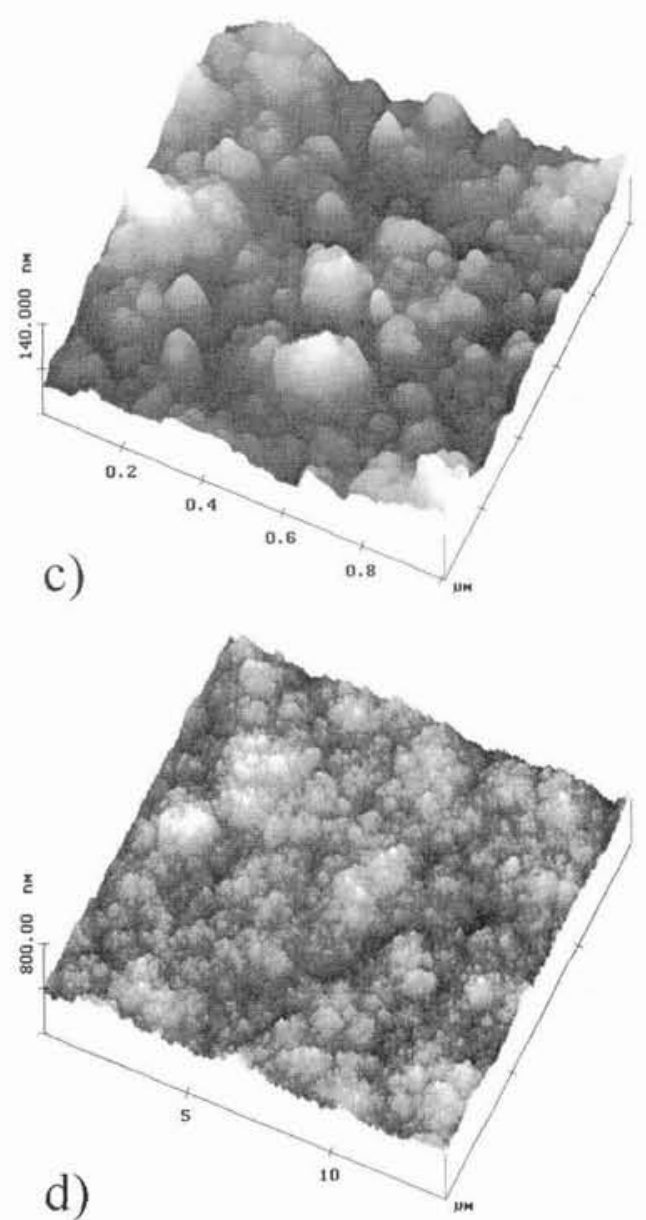

Figure 1. AFM micrographs of $\mathrm{SiO}_{2}$ films grown for 1 and 30 hours at $611 \mathrm{~K}(\mathrm{a}, \mathrm{b})$ and $723 \mathrm{~K}(\mathrm{c}, \mathrm{d})$. Note the different areas scanned for the $1 \mathrm{~h}(\mathrm{a}, \mathrm{c})$ and $30 \mathrm{~h}(\mathrm{~b}, \mathrm{~d})$ films.

a) $611 \mathrm{~K}$

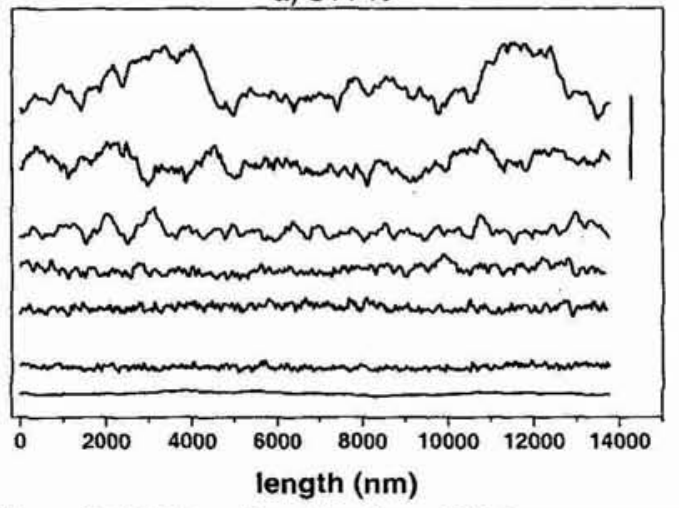

b) $723 \mathrm{~K}$

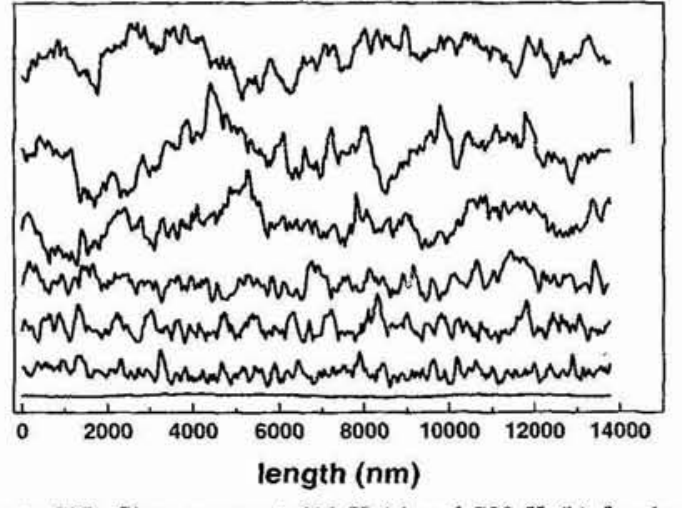

Figure 2. Height profiles taken from AFM images corresponding to $\mathrm{SiO}_{2}$ films grown at $611 \mathrm{~K}$ (a) and $723 \mathrm{~K}$ (b) for the following times (from bottom to top): 0 (bare substrate), $35,60,180,480,900$ and $2880 \mathrm{~min}$. The vertical bar corresponds to $100 \mathrm{~nm}$. 


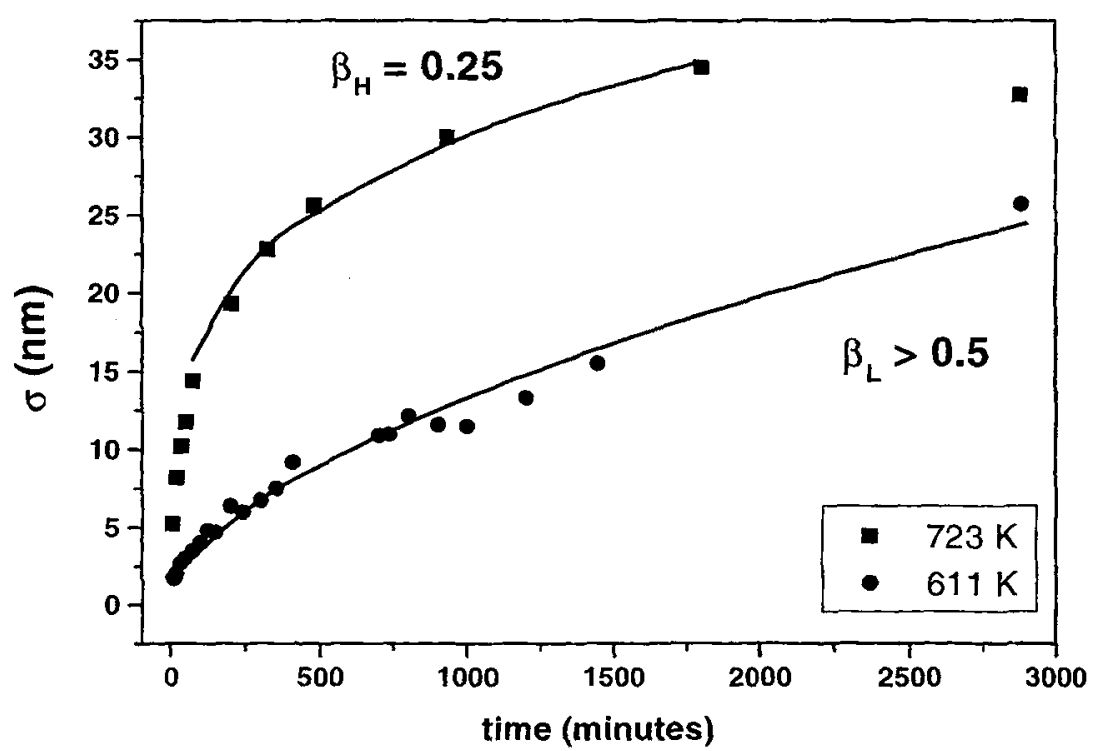

Figure 3. Temporal evolution of the saturated surface roughness for films grown at $611 \mathrm{~K}$ and $723 \mathrm{~K}$.

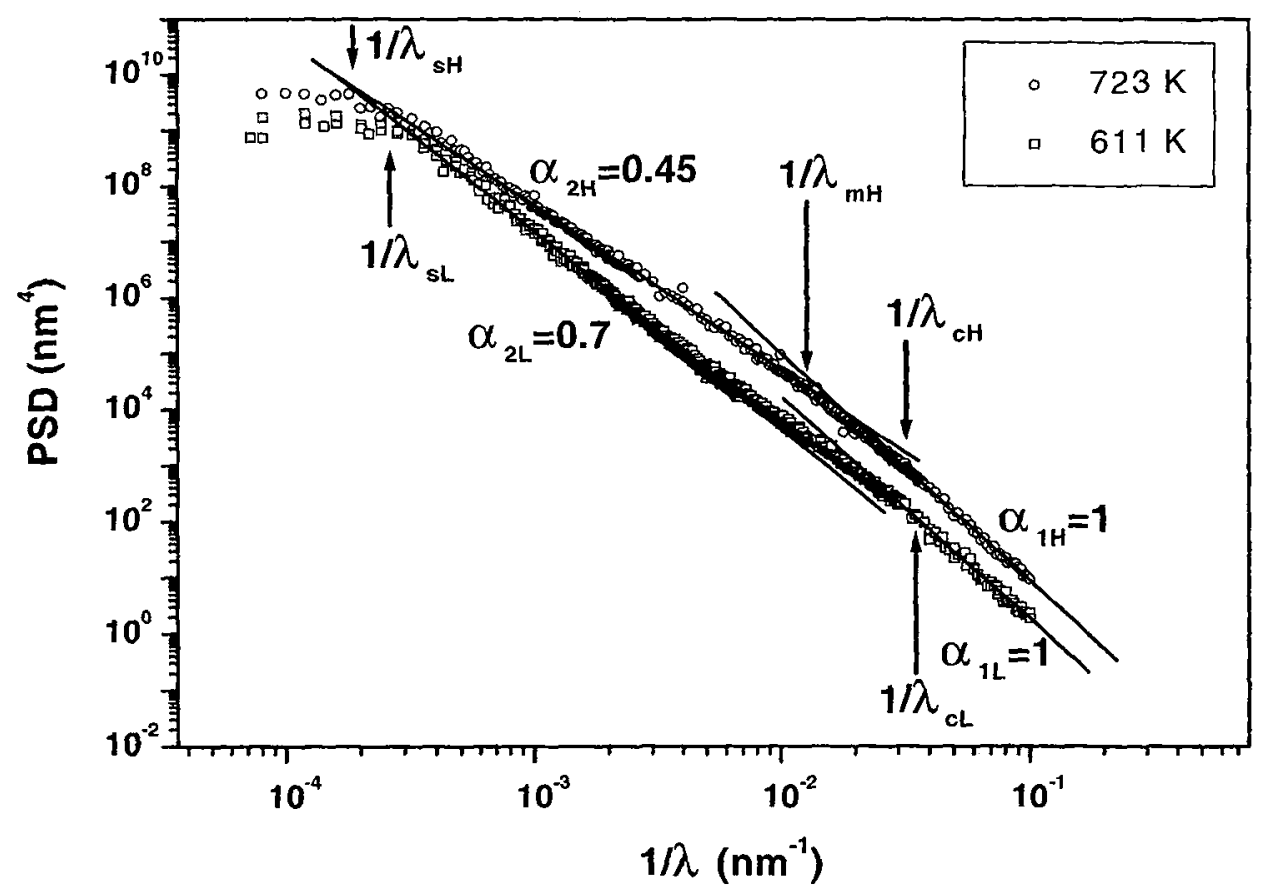

Figure 4. PSD functions obtained from AFM images corresponding to $\mathrm{SiO}_{2}$ films grown for $48 \mathrm{~h}$ at $611 \mathrm{~K}$ and $723 \mathrm{~K}$. 



Figure 5. PSD temporal evolution for $\mathrm{SiO}_{2}$ films grown at $611 \mathrm{~K}$ (a) and $723 \mathrm{~K}$ (b) at different wavelengths: $20 \mathrm{~nm}(\mathrm{D}), 0.1$ $\mu \mathrm{m}(\mathrm{O}), 0.2 \mu \mathrm{m}(\Delta), 0.5 \mu \mathrm{m}(\nabla), 1 \mu \mathrm{m}(0), 10 \mu \mathrm{m}(+)$ and $14.2 \mu \mathrm{m}(\times$, only for $723 \mathrm{~K})$.

indicates that the roughness is saturated. Below the saturation wavelengths, either $\lambda_{\text {sL }}$ or $\lambda_{\text {sH }}$, different regions can be distinguished for the value of $\alpha$. At low temperature, we obtain $\alpha=1$ for $\lambda<\lambda_{\text {cL }}$ and $\alpha \approx$ 0.7 for $\lambda_{\mathrm{cL}}<\lambda<\lambda_{\mathrm{sL}}$. At high temperature, there is an additional region with $\alpha \approx 0.45$ for $\lambda_{\mathrm{mH}}<\lambda<\lambda_{\mathrm{sH}}$. Analogously, we obtain $\alpha$ equal to 1 for $\lambda<\lambda_{c H}$.

A more detailed study of the roughness evolution with deposition time can be achieved through examination of the PSD intensity at different wavelengths. Figure 5 shows the temporal evolution of the PSD intensity, at wavelengths ranging from $20 \mathrm{~nm}$ up to $14.2 \mu \mathrm{m}$, for low (a) and high (b) temperatures. In both cases, all Fourier modes exhibit an initial increase and finally become stable above a certain deposition time, in such a way that the shorter the frequency of the mode, the sooner it becomes stable. The main difference lies in the fact that at low temperature there are unstable modes during all deposition times, whereas at high temperature all modes have become stable above $15 \mathrm{~h}$ of deposition.

\section{DISCUSSION}

As shown in the previous section, the most important difference between the low and high temperature series concerns the growth stability found at high temperature. From the $\alpha$ and $\beta$ values it is possible to determine the responsible growth mechanism for this stability. Among the mechanisms that tend to stabilise the growth of a solid growing film, we should consider surface diffusion [13], adsorption/desorption [14], and non-linear lateral growth as described by Kardar, Parisi and Zhang (KPZ growth) [15]. These mechanisms are characterised by the exponents $\alpha=1$ and $\beta=0.25, \alpha=\beta=0$, and $\alpha$ $\approx 0.4$ and $\beta \approx 0.25$, respectively $[12,16]$.

At both temperatures and for short wavelengths $\left(\lambda<\lambda_{\mathrm{cL}, \mathrm{H}}\right)$, we have found $\alpha=1$, which indicates that morphology evolution is dominated by surface diffusion at short length scales [17]. For larger wavelengths there is a region with $\alpha=0.7$ and $\beta>0.5$ in both cases, due to instabilities inherent to the CVD process related to the presence of concentration gradients in the gas-phase $(\beta=0.5$ corresponds just to random deposition) [12]. This kind of instabilities corresponds to preferential growth at the prominent zones of the film surface (tips) with detriment to growth at the depressed ones (valleys) [18]. At high temperature, the PSD function shows a region with $\alpha \approx 0.45$, a value much closer to the 0.4 value predicted for the KPZ growth than to any other stabilising mechanism, as mentioned above. This region becomes larger with time, and for the 48 hours deposited sample it almost extends up to 2 decades in length. Therefore, we propose that the stable growth found at high temperature corresponds to the existence of a KPZ-type mechanism. The observation of a region with $\beta \approx 0.25$ in Figure 3 just before reaching saturation supports this hypothesis. It remains open to discussion whether or not a similar KPZ region could be found for the low temperature samples for much longer deposition times (i.e., more than 2 
days!). This possibility is suggested by the existence, previously to the KPZ region $\left(\lambda_{\mathrm{mH}}>\lambda>\lambda_{\mathrm{cH}}\right)$, in Figure 4 of an unstable region. However, only experimental verification could give us a reliable answer to this question.

The physical origin of the KPZ growth can be explained in terms of the lower sticking coefficient in comparison with the low temperature growth. In the work of Watanabe and Komiyama [19], sticking coefficients, as obtained by the micro/macro cavity method, of 0.08 and 0.4 at 723 and $611 \mathrm{~K}$, respectively, were reported for the $\mathrm{SiH}_{4} / \mathrm{O}_{2}$ system. These results are in agreement with the unstable/stable transition observed by us when increasing temperature from 611 to $723 \mathrm{~K}$. A low sticking coefficient means that impinging precursor species are able to sample many surface sites before they become finally incorporated to the growing interface. In this sense, as the sticking coefficient is lower the different growing sites on the film surface acquire similar probabilities of being sampled. Thus, lateral growth would be favoured, which would counteract the reactant depletion promoted by concentration gradients and finally would stabilise the film surface. This result is consistent with the qualitative experimental and theoretical findings for conformal growth of CVD silica under our experimental conditions [19]. It is found that, when conformal growth takes place on the substrate trenches, lateral growth (in particular on the wall trenches) is promoted, which is the signature of $\mathrm{KPZ}$ growth $[12,15,20]$. This is just the case when the substrate temperature is increased.

The observed evolution of the PSD intensity at different wavelengths disagrees with predictions of linear stability analysis [21-26], from which we would expect all modes below a certain wavelength to be stable and all others above to be unstable. Although this can be explained by the presence of the non-linear KPZ regime, agreement is reached regarding the rate at which different modes become stable, in the sense that stability is achieved earlier for shorter wavelengths.

However, it is worth to comment on the discrepancy obtained between the experimental data and those derived from the theoretical models. From the experimental study it is observed that the saturation region for the high temperature samples appears clearly after 15 hours of growth. Also, if saturation were present for the low temperature set (a question that is not evident), it would have to appear after 2 days of deposition. This fact could imply that the asymptotic growth regime has neither been achieved in the theoretical simulations so far, which, because of computational limitations, would be restricted to the first stages of the growth process. This limitation was already formerly addressed in previous theoretical approaches to the CVD growth process $[18,23,24]$.

The temporal evolution of PSD at different wavelengths indicates that surface diffusion, which operates within a short length scale, defines rapidly the film structure at the nanometric level, a feature of vital importance in the resulting film density. In particular, surface diffusion dominates the surface morphology for length scales smaller than the typical grain size. By contrast, KPZ growth regime, which operates over large length scales, takes a long time before becoming triggered. Probably, this explains the scarce number of experimental observations of $\mathrm{KPZ}$ growth. In our system, KPZ growth likely accounts for the reemission of impinging species, which is enhanced by low sticking coefficients, providing latera] zones of the film surface with additional precursor species over those provided by direct mass flux from the gas-phase. Reemission has been reported to be the most relevant mechanism in the production of conformal films over micro-sized trenches $[9,10]$ and even over cavity walls hidden to direct mass flux [27].

\section{CONCLUSIONS}

The growth evolution of CVD silica films has been studied for low and high temperatures. It has been found that at $611 \mathrm{~K}$ the surface is unstable, whereas at high temperature $(723 \mathrm{~K})$ it finally reaches a saturation regime. This means that, under the latter conditions, the growth is stable, which is an interesting result from both the theoretical and experimental points of view. For the films grown at $723 \mathrm{~K}$ it is seen that the main stabilising mechanism is the KPZ term which accounts for the lateral growth of the surface structures. This lateral growth is enhanced, in this system, by the decrease of the sticking coefficient with temperature, as it is qualitatively observed in the conformal growth of CVD silica on substrate trenches [20]. It is proposed that, because of the reemission processes of the incoming particles from the growing interface, these particles can be finally incorporated to surface sites that are quite unlikely to be reached in case that these reemission processes do not take place (i.e., for higher sticking 
coefficients). Finally the experimental data are contrasted with those obtained from theoretical simulations. Their differences are explained on the basis of the difficulty to reach the asymptotic growth regimes in the standard theoretical studies of CVD growth.

\section{Acknowledgements}

This work was partially realized within the frame of the CONICET-CSIC research cooperation program, partially supported by Ministerio de Educación y Cultura (Programa de Cooperación Científica con Iberoamérica), by the 7220 -ED/082 project from ECSC and by the 07M/0710/97 project from CAM. F. Ojeda gratefully acknowledges the grant financed by the Comunidad de Madrid (683/96, BOCM 22/4/96).

\section{References}

[1] Vasilyeva L.L., Drozdov V.N., Repinsky S.M., Svitashev K.K., Thin Solid Films 55 (1978) 221-228.

[2] Ojeda F., Castro-García A., Gómez-Aleixandre C., Albella J.M., J. Mater. Res. 13 (1998) 2308-2314.

[3] Becker F.S., Pawlik D., Anzinger H., Spitzer A., J. Vac. Sci. Tech. B 5 (1987) 1555-1563.

[4] Takahashi T., Hagiwara K., Egashira Y., Komiyama H., J. Electrochem. Soc. 143 (1996) 1355-1361.

[5] Nguyen S., Dobuzinsky D., Harmon D., Gleason R., Fridmann S., J. Electrochem. Soc. 137 (1990) 2209-2215.

[6] Fry H.W., West J.P., Poon S., Boeck B.A., Yu C.C., Solid State Tech. March (1994) 31-40.

[7] Bennet B.R., Lorenzo J.P., Vaccaro K., Appl. Phys. Lett. 50 (1987) 197-199.

[8] Mitchener J.C., Mahawili I., Solid State Tech. August (1987) 109-1 13.

[9] Cooke M.J., Harris G., J. Vac. Sci. Tech. A 7 (1989) 3217-3221.

[10] Coronell D.G., Jensen K.F., J. Electrochem. Soc. 141 (1994) 2545-2551.

[11] Stout P.J., Kushner M.J., J. Vac. Sci. Tech. A 11 (1993) 2562-2571.

[12] Barabasi A.-L., Stanley H.E., Fractal concepts in surface growth (Cambridge University press, 1995).

[13] Wolf D.E., Villain J., Europhys. Lett. 13 (1990) 389-394.

[14] Edwards S.F., Wilkinson D.R., Proc. R. Soc.London A 381 (1982) 17-31.

[15] Kardar M., Parisi, Zhang Y.C., Phys. Rev. Lett. 56 (1986) 889-892.

[16] Tong W.M, Williams R.S, Annu. Rev. Phys. Chem. 45 (1994) 401-438.

[17] Vázquez L., Salvarezza R.C., Herrasti P., Ocón P., Vara J.M., Arvia A.J., Phys. Rev. B 52 (1995) 2032-2037.

[18] Bales G.S., Redfield A.C., Zangwill A., Phys. Rev. Lett. 62 (1989) 776-779.

[19] Watanabe K., Komiyama H., J. Electrochem. Soc. 137 (1990) 1222-1227.

[20] Carter G., in Erosion and growth of solids stimulated by atom and ion beams, Kiriakidis G., Carter G, Whitton J.L. (Eds.) (Martinus nijhoff, Dordrecht, 1986), and references therein, pp. 70-97.

[21] Van den Brekel C.H.J, Jansen A.K., J. Crystal Growth 43 (1978) 364-370.

[22] Jansen A.K., van den Brekel C.H.J., J. Crystal Growth 43 (1978) 371-377.

[23] Paimer B.J., Gordon R.G., Thin Solid Films 158 (1988) 313-341.

[24] Palmer B.J., Gordon R.G., Thin Solid Films 177 (1989) 141-159.

[25] Ananth R., Gill W.N., J. Crystal Growth 118 (1992) 60-70.

[26] Viljoen H.J., Thiart J.J., Hlavacek V., AIChE J. 40 (1994) 1032-1045.

[27] Cheng L.-Y., McVittie P., Saraswat K.C., Appl. Phys. Lett. 58 (1991) 2147-2149. 